\title{
Frequency and fitness cost of resistance to Bacillus thuringiensis in Chrysomela tremulae (Coleoptera: Chrysomelidae)
}

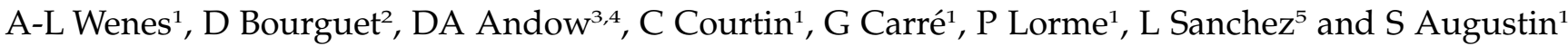 \\ ${ }^{1}$ Institut National de la Recherche Agronomique, Centre de Recherches d'Orléans, Unité de Zoologie Forestière, Ardon, Olivet 45166, \\ France; ${ }^{2}$ Institut National de la Recherche Agronomique, UMR Centre de Biologie et de Gestion des Populations (CBGP), Campus \\ International de Baillarguet, Montferrier-sur-Lez 34988, France; ${ }^{3}$ Department of Entomology, University of Minnesota, St Paul, MN \\ 55108, USA; ${ }^{4}$ Minnesota Center for Community Genetics, University of Minnesota, St Paul, MN 55108, USA; ${ }^{5}$ Institut National de la \\ Recherche Agronomique, Centre de Recherches d'Orléans, Unité Amélioration, Génétique et Physiologie Forestière, Ardon, Olivet 45166, \\ France
}

The 'high dose-refuge' (HDR) strategy is commonly recommended and currently used for delaying or preventing pest adaptation to transgenic plants producing Bacillus thuringiensis $(B t)$ toxins. The efficiency of this strategy depends, among other factors, on the initial frequency of $B t$ resistance alleles and on the fitness costs associated with these alleles. Two years ago, an allele conferring resistance to Bt poplar was detected in a French population of the poplar pest Chrysomela tremulae F. Although this pest had never been subjected to $B t$ selection pressure due to human activities, the frequency of this allele was estimated at 0.0037 , with a $95 \%$ credible $(\mathrm{Cl})$ interval of $0.00045-0.0080$. We investigated the frequency of this allele in a second sample of $C$. tremulae collected more than $500 \mathrm{~km}$ from the site of the initial population. The estimated frequency in this sample was $0.0113(95 \% \mathrm{Cl} 0.0031-0.0247)$, reinforcing the conclusion that resistance to $B t$ plants may be present at detectable frequencies in pest populations before selection resulting from pest management by humans. The frequency of the Bt resistance allele over the two samples was 0.0049 (95\% Cl $0.0020-0.0091)$. We also followed five laboratory lines in which the frequency of this allele was initially fixed at 0.500. After five generations maintained on non-Bt poplar leaves, the frequency of this allele decreased in all lines, whereas allelic frequencies at a neutral locus were unaffected. Thus, the $B t$ resistance allele detected in French populations of $C$. tremulae is probably associated with a fitness cost.

Heredity (2006) 97, 127-134. doi:10.1038/sj.hdy.6800845; published online 17 May 2006

Keywords: transgenic poplar; Bacillus thuringiensis; Chrysomela tremulae; resistance allele frequency; fitness cost; resistance management

\section{Introduction}

Transgenic plants producing Bacillus thuringiensis $(B t)$ toxins to control their key pests provide an attractive alternative to conventional insecticide sprays (Shelton et al, 2002; Carrière et al, 2003). One of the main risks associated with the widespread use of these plants is rapid adaptation of the pest targeted by the toxins (Gould, 1998). Field resistance to Bt cotton has been reported for very few moth species feeding on this crop, but field resistance to $B t$ toxin sprays has been detected in several populations of Plutella xylostella and many targeted pests have been selected for $B t$ resistance in the laboratory (reviews in Ferré and Van Rie, 2002; Tabashnik et al, 2003; Griffits and Aroian, 2005). The marketing of $B t$ plants has therefore generated concern about the evolution of resistance in field populations, leading to theoretical and empirical studies of effective resistance management strategies.

Correspondence: S Augustin, Institut National de la Recherche Agronomique, Centre de Recherches d'Orléans, Unité de Zoologie Forestière, Ardon, Olivet 45166, France. E-mail: augustin@orleans.inra.fr Received 8 November 2005; accepted 11 April 2006; published online 17 May 2006
The most commonly used system for delaying or preventing pest adaptation to $B t$ crops is the "high dose-refuge' (HDR) strategy, originally proposed by Georghiou and Taylor (1977) for managing the evolution of resistance to conventional insecticides. It involves the planting of non- $B t$ host plant refuges close to fields of $B t$ crop, to promote the survival of susceptible pests (Alstad and Andow, 1995). In the HDR strategy, as in all resistance management strategies, the time to control failure - defined as the number of generations or years before the frequency of resistance reaches $50 \%$ - strongly depends on factors such as the initial frequency of $B t$ resistance alleles, the degree of dominance of the resistance conferred by these alleles, and the gene flow within and between populations. Among these factors, the frequency is certainly one of the most important: the lower the frequency, the more sustainable the strategy. The initial frequency of resistance has been estimated in only a limited number of cases, mostly in lepidopteran pests, due to practical limitations: Helicoverpa armigera (Wu et al, 2002), Heliothis virescens (Gould et al, 1997) and H. zea (Burd et al, 2003) on cotton, Ostrinia nubilalis on maize (Andow et al, 1998, 2000; Bourguet et al, 2003), Scirpophaga incertulas (Bentur et al, 2000) on rice, and 
Pectinophora gossypiella (Tabashnik et al, 2000) on cotton. These studies yielded contrasting results, with the frequency of $B t$ resistance alleles ranging from $10^{-4}$ to $10^{-1}$.

Another factor influencing the evolution of resistance is the fitness cost - the decrease in fitness associated with the resistance gene in the absence of selective pressure associated with some pesticide resistance alleles (Coustau et al, 2000). Indeed, if resistance entails a fitness cost, the spread of a recessive allele could be prevented by an appropriate resistance management strategy (Lenormand and Raymond, 1998; Carrière and Tabashnik, 2001; Carrière et al, 2004; Bates et al, 2005). Although fitness costs associated with $B t$ resistance were not always detected (eg Gould and Anderson, 1991; Tang et al 1997), they have been reported in many pests (Groeters et al, 1994; Trisyono and Whalon, 1997; Alyokhin and Ferro, 1999; Oppert et al, 2000; Carrière et al, 2001a,b; Akhurst et al, 2003; Janmaat and Myers, 2003; Higginson et al, 2005. Bird and Akhurst (2004, 2005) showed that, in $H$. armigera, most fitness costs associated with $B t$ resistance (eg a lower percentage of survival or a longer mean time to pupation) are recessive.

Chrysomela tremulae Fabricius (Coleoptera, Chrysomelidae) is a pest of native and introduced hybrid poplars in France (Augustin and Lévieux, 1993). In a previous investigation, we showed that an allele conferring resistance to $B t$ poplar in a field population of $C$. tremulae of the Centre region of France was segregating at a frequency of 0.0037 (Génissel et al, 2003a). We subsequently demonstrated that this resistance was completely recessive and determined by a single allele at one autosomal locus (Augustin et al, 2004).

The principal aim of this study was to determine whether the estimated frequency of resistance in the Centre region of France was a general feature of natural populations of $C$. tremulae. We sampled feral beetles from a poplar plantation located more than $500 \mathrm{~km}$ from the site from which the sample collected by Génissel et al (2003a) was taken. Individual beetles were crossed with adults homozygous for the $B t$ resistance allele detected by Génissel et al (2003a). The secondary aim of this study was to determine whether this $B t$ resistance allele was associated with a fitness cost. We compared changes in the frequency of the $B t$ resistance allele and neutral alleles in five lines seeded with heterozygous $R S$ resulting from a mass-crossing between a susceptible strain and a resistant strain.

\section{Materials and methods}

\section{Susceptible and resistant strains of $C$. tremulae}

We screened for $B t$ resistance alleles and carried out fitness cost experiments with three - one susceptible and two resistant - strains of $C$. tremulae. The susceptible (S\#125) strain originated from the offspring of an isofemale line displaying no resistance to the Cry3Aa toxin (Génissel et al, 2003a). The two resistant strains, R\#60 and R\#116, were established from two different resistant isofemale lines selected on the foliage of a transgenic $B t$ poplar line producing the Cry3Aa Bt toxin (Génissel et al, 2003a). Both the R\#60 and R\#116 strains are fixed for a recessive allele conferring resistance to the Cry3Aa toxin (Augustin et al, 2004; Augustin, unpublished data).
Hence, all beetles from these strains had resistant homozygous $R R$ genotypes whereas those from the S\#125 strain had susceptible homozygous SS genotypes. These three strains were maintained in standard rearing conditions, in a growth chamber at $20^{\circ} \mathrm{C}$ with a photoperiod of 16:8 (L:D). Larvae and adults of the susceptible strain were fed on fresh mature leaves of non-Bt poplars. Neonate larvae of the two resistant strains were fed on fresh mature leaves of the $B t$ poplar line described below and were then transferred onto non-transgenic foliage.

\section{Screening for $B t$ resistance}

We screened for resistance by feeding F1 neonates on leaf discs cut from the fresh mature leaves of a $B t$ poplar line placed on moist filter paper. This $B t$ poplar line is a hybrid clone (Populus tremula $\times P$. tremuloides, Institut de la Recherche Agronomique No. 353-38) expressing a synthetic cry $3 \mathrm{Aa}$ gene from Bacillus thuringiensis spp tenebrionis (Génissel et al, 2003b). Its leaves contained enough Cry3Aa toxin to kill susceptible homozygous and heterozygous $C$. tremulae in less than 3 days (Augustin et al, 2004). Thus, all larvae that had fed actively on $B t$ poplar and survived for 4 days or more were classified as resistant.

\section{Frequency of $B t$ resistance in a natural population}

Insect sampling: Feral insects were sampled at a single site at Bar-le-Duc in the Lorraine region of France. At this site, two groups of adults were collected on young leaves and twigs of $P$. trichocarpa poplars in June and July 2003. The probability of sampling sibling individuals was minimised by sampling adults uniformly over the entire area (ca 7 ha) of the field. The sex of each adult was determined and each female was caged alone to check her mating status: mated or virgin. All males and virgin females were screened for resistance to $B t$ poplar.

Screening procedure: Feral males and virgin females were mated individually with virgin females and males of the R\#60 strain. Eggs masses were collected daily and F1 neonates emerging from these masses were screened for $B t$ resistance as described above. As the parents of the R\#60 strain insects were $R R$, we expected $50 \%$ of the F1 individuals to be $R R$ if the feral parent was $R S$. Neonates of each line were also tested on non- $B t$ foliage to estimate $\mu$, the probability that the individual died for reasons other than susceptibility to the toxin.

Data analysis: Bayesian methods were used to calculate the expected frequency of resistance and the probability of not detecting a resistance allele in the experiment, extending the methods described by Andow and Alstad (1998) and Stodola and Andow (2004). Frequentist statistics can be used on these data (Gould et al, 1997), but Bayesian methods have the advantage of explicitly taking into account sample size. Bayesian statistics have several additional advantages over frequentist statistics for resistance. Bayesian methods use all available information, as we shall show in this paper, while frequentist measures are specific to the particular experiment. Bayesian methods depend only on probabilities of results that actually did occur, while frequentist measures incorporate probabilities of 'data' that were possible, but did not actually occur. Most importantly, 
Bayesian measures are tailored to decision analysis, while frequentist measures generally are not (Brunk, 1975).

Let $S$ be the number of lines testing positive for resistance, $N$ the total number of lines tested, $p$ the frequency of resistant lines, $\hat{p}$ the estimated frequency of resistant lines, $q$ the frequency of resistance alleles, $\hat{q}$ the estimated frequency of resistance alleles, $u$ and $v$ are the parameters of the prior beta distribution of $p(u=v=1$ for the uninformative uniform prior distribution), $J$ the number of $F_{1}$ larvae screened in each line, $\mu$ the mortality of $F_{1}$ larvae unrelated to toxin susceptibility, and $N_{R}$ the number of $F_{1}$ larvae testing positive in each line.

As $S$ is binomially distributed $(N, p)$ and the prior distribution of $p$ is $\operatorname{bet} a(u, v)$, the posterior distribution of $p$ is given by $g(p \mid S)=\operatorname{beta}(S+u, N-S+v)$, which is identical to equation (14) from Andow and Alstad (1999). The expected value of $p$ is

$$
\hat{p}=E[p]=\frac{S+u}{N+u+v}
$$

The $95 \%$ confidence intervals for $\hat{p}$ were calculated according to equation (15) from Andow and Alstad (1999), using Mathematica (Wolfram Research Inc. 1999).

As this resistance is recessive and determined by a single allele at one autosomal locus (Augustin et al, 2004), $\hat{q}$ and its $95 \%$ credible interval were estimated as follows. Assuming that the sample was from a panmictic population, the frequency of $R R$ should be $q^{2}$ and the frequency of $R S$ should be $2 q(1-q)$. This implies that $p=q^{2}+2 q(1-q)$, and thus, by rearrangement, application of the quadratic formula, simplification and selection of the only feasible root:

$$
\hat{q}=1-(1-\hat{p})^{1 / 2}
$$

The $95 \%$ credibility intervals of $\hat{q}$ can also be calculated using equation (2), from the $95 \%$ credible intervals for $\hat{p}$. Allele frequency estimates can be compared from different samples. Let $u_{1}, v_{1}$ and $q_{1}$ be from the first sample and $u_{2}, v_{2}$ and $q_{2}$ be from the second sample. Assuming that the samples are independent, we use the joint likelihood ratio statistic, $W\left(q_{1}, q_{2}\right)$, which is based on the posterior distributions of $q_{1}$ and $q_{2}$, and calculate the joint 95\% credible region around $\hat{q}_{1}$ and $\hat{q}_{2}$, using standard methods. If the credible region overlaps the line $q_{1}=q_{2}$, then the sample estimates are not significantly different. If the credible region does not overlap the line $q_{1}=q_{2}$, then the sample estimates are different. We compared the allele frequency estimate from Génissel et al (2003a) with the estimate observed here. The joint log-likelihood function for this comparison is

$$
\begin{aligned}
l\left(q_{1}, q_{2}\right)= & u_{1} \ln \left(1-\left(1-q_{1}\right)^{4}\right)+v_{1} \ln \left(\left(1-q_{1}\right)^{4}\right) \\
& +u_{2} \ln \left(1-\left(1-q_{2}\right)^{2}\right)+v_{2} \ln \left(\left(1-q_{2}\right)^{2}\right)
\end{aligned}
$$

where the subscript 1 is for the data from Génissel et al (2003a) and the subscript 2 is for the data from this paper. According to standard methods, the joint likelihood ratio statistic is

$$
W\left(q_{1}, q_{2}\right)=2\left(l\left(\hat{q}_{1}, \hat{q}_{2}\right)-l\left(q_{1}, q_{2}\right)\right)
$$

Data for the number of $F_{1}$ individuals screened for each line was used to estimate the probability that resistance was missed $\left(P_{N o}\right)$. For each line, $P_{N o}$ is equal to the prob- ability that no $R R \mathrm{~F}_{1}$ individuals were successfully identified by screening, given that $J$ individuals were screened and $\mu$ is the probability that the individual died for reasons other than susceptibility to the toxin. The probability of an $\mathrm{F}_{1}$ individual having an $R R$ genotype is $1 / 2$, so

$$
\begin{aligned}
& \mu=0 \quad P_{N o}=\left(\begin{array}{l}
J \\
0
\end{array}\right)(1 / 2)^{J} \\
& \mu \neq 0 \quad P_{N o}=\sum_{k=0}^{J} \mu^{k}\left(\begin{array}{l}
J \\
k
\end{array}\right)(1 / 2)^{J}
\end{aligned}
$$

$\mu$ was estimated from the lines testing positive for resistance. Although $\mu$ is defined as the probability that an $F_{1}$ individual died for reasons other than that tested in the screen (ie susceptibility to the toxin), the only individuals of importance are the $R R \mathrm{~F}_{1}$ homozygotes, so $\mu$ equates to be the probability that an $R R \mathrm{~F}_{1}$ larvae died for reasons other than susceptibility to the toxin. Larvae may die for many reasons, including handling, fitness costs of resistance and poor adaptation to the test conditions. Let $k$ be the number of $R R \mathrm{~F}_{1}$ larvae among the $J$ larvae screened, and $P_{k \mid J}=\operatorname{Prob}\left(k R R\right.$ larvae $\mid J \mathrm{~F}_{1}$ larvae screened). For each line testing positive, $N_{R} F_{1}$ larvae survived the screen $\left(N_{R}>0\right)$. Let $P_{k \mid J, N_{R}}=$ Prob ( $k R R$ larvae I $J \mathrm{~F}_{1}$ larvae screened) and $N_{R}$ the number of $\mathrm{F}_{1}$ larvae testing positive. For $P_{k \mid J}, N_{R}$, it is clear that $k \geq N_{R}$ (there must be at least as many $R R$ larvae tested, $k$ as the number of $R R$ larvae testing positive, $N_{R}$ ). From the laws of total probability and conditional probability

$$
\begin{aligned}
E\left[\mu \mid J, N_{R}\right] & =\sum_{k=R}^{J} E\left(\mu \mid k, N_{R}\right) P_{k \mid J, N_{R}} \\
& =\sum_{k=N_{R}}^{J} E\left(\mu \mid k, N_{R}\right) \frac{P_{k \mid J}}{\sum_{k=N_{R}}^{J} P_{k \mid J}}
\end{aligned}
$$

where

$$
P_{k \mid J}=\left(\begin{array}{c}
J \\
K
\end{array}\right)(1 / 2)^{J}
$$

and the expected value of $\mu$ given $k$ and $R$ follows from equation (1) and is $E\left(\mu \mid k, N_{R}\right)=1-\left(N_{R}+1\right) /(k+2)$.

$P_{N o}$ was calculated from the estimated $\mu$ and equation (5) for each of the lines in which no resistance was detected. The experiment-wise probability of a single additional false negative line is the mean $P_{N o}$ calculated for each line.

\section{Fitness cost}

Experimental setting: Heterozygous $R S$ individuals referred to as the F0 individuals - were produced by mass-crossing the susceptible (S\#125) and resistant (R\#60) strains and used to set up five independent lines. Each line was founded by 15 females and 10 males providing about 250-700 F1 RS offspring. For each line, we established 20 mating pairs of F1 adults in individual boxes. A male and a virgin female were randomly selected from the F2 offspring of each mating pair and randomly mated, to give 20 mating pairs of F2 adults. The same procedure was applied to obtain the F3, F4 and F5 generations.

Allelic frequency at the resistance locus: For each line, the frequency of the $B t$ resistance allele was estimated at 
generations F3 and F5. For this purpose, virgin adults were randomly selected from the offspring of the F2 and F4 mating pairs, respectively. Each of these adults was individually mated with a virgin beetle of the R\#116 strain. Egg masses were collected from each mating pair daily and F1 neonates emerging from these masses were screened for $B t$ resistance, as described above. After correcting for mortality rates on non-Bt foliage, we expected 0,50 and $100 \%$ mortality when the tested F3 or F5 parents were $S S, R S$ and $R R$, respectively.

Allelic frequency at the $C k$ loci: For each line, F0 individuals and the F3 and F5 adults used to estimate $B t$ resistance allele frequency were also genotyped at the creatine kinase $(C k)$ locus, by starch gel electrophoresis. The abdomen of each individual was crushed in $100 \mu \mathrm{l}$ of $0.4 \%$ NADP Tris-citrate $\mathrm{pH} 6.7$ buffer. The homogenates were subjected to horizontal starch gel electrophoresis in the Tris-citrate pH 6.7 buffer system (Pasteur et al, 1987), with CK (EC 2.7.3.2) detection as described by Génissel et al (2000).

Data analysis: For each line, the allelic frequencies and their $95 \% \mathrm{CI}$ - at the resistance and $C k$ loci were calculated, using the Bayesian methods described above. Tests for deviations from Hardy-Weinberg equilibrium at each locus and for genotypic linkage disequilibrium between the resistance and $C K$ loci were carried out for each individual line and for all the lines considered together, with GENEPOP 3.4. (Raymond and Rousset, 1995).

\section{Results}

Frequency of $B t$ resistance alleles in a field population We carried out a total of 286 crosses between individuals collected at Bar-le-Duc and individuals of the resistant R\#60 strain: 192 of these crosses were feral male $\times$ R\#60 female crosses and 94 were feral female $\times$ R\#60 males crosses. We found that 102 of the lines were sterile and eight lines produced too few offspring (ie $<5$ larvae) for analysis. Three of the remaining 176 lines screened for Bt resistance had resistant F1 larvae. The estimated frequency of resistance was 0.0113 , with a $95 \%$ credible interval of $0.0031-0.0247$.

The proportions of resistant larvae in the three positive lines were $14.3(N=28), 40.8(N=184)$ and $8.9 \%(N=56)$. From these data, we calculated a weighed average, $\mu=0.3599$, corresponding to the experiment-wise probability of a false negative among the lines testing negative. The probability of a false negative was $P_{N o}=5 \times 10^{-3}$.

\section{Fitness cost}

Two alleles, $C k 80$ and $C k 100$, were detected at the $C k$ locus. Exact tests for genotypic linkage disequilibrium between this locus and the resistance locus were not significant for any of the lines tested individually $(P>0.12$ for all lines) or for all lines considered together $(P=0.845)$. Changes in allelic frequencies at the resistance and $C k$ loci over generations may therefore be considered independent.

The estimated frequencies of the $R$ and $C k 80$ alleles at generations F0, F3 and F5 are given in Table 1. The frequency of the $R$ allele decreased over generations, in

Table 1 Estimated frequencies of the $R(q R)$ and $C k 80(q C k 80)$ alleles and their $95 \% \mathrm{CI}$ within each line and for all lines considered together at generations F0, F3 and F5

\begin{tabular}{|c|c|c|c|c|c|c|c|c|}
\hline & \multicolumn{8}{|c|}{ Locus } \\
\hline & \multicolumn{4}{|c|}{ Resistance } & \multicolumn{4}{|c|}{$\mathrm{Ck}$} \\
\hline & $\mathrm{N}^{\mathrm{a}}$ & $\# R^{b}$ & $\mathrm{qR}$ & $95 \% C I$ & $\mathrm{~N}^{\mathrm{a}}$ & $\# \mathrm{Ck} 80^{\mathrm{c}}$ & qCk80 & $95 \% C I$ \\
\hline \multicolumn{9}{|c|}{ Generation F0 } \\
\hline Line \#1 & - & & 0.500 & - & 19 & 3 & 0.100 & {$[0.027,0.209]$} \\
\hline Line \#2 & - & & 0.500 & - & 23 & 4 & 0.104 & {$[0.035,0.204]$} \\
\hline Line \#3 & - & & 0.500 & - & 19 & 5 & 0.150 & {$[0.059,0.274]$} \\
\hline Line \#4 & - & & 0.500 & - & 19 & 2 & 0.075 & {$[0.016,0.173]$} \\
\hline Line \#5 & - & & 0.500 & - & 16 & 2 & 0.088 & {$[0.019,0.202]$} \\
\hline All lines & - & & 0.500 & - & 96 & 16 & 0.087 & {$[0.052,0.131]$} \\
\hline \multicolumn{9}{|c|}{ Generation F3 } \\
\hline Line \#1 & 13 & 8 & 0.321 & {$[0.165,0.502]$} & 17 & 13 & 0.389 & {$[0.239,0.551]$} \\
\hline Line \#2 & 13 & 7 & 0.286 & {$[0.138,0.463]$} & 22 & 3 & 0.087 & {$[0.025,0.183]$} \\
\hline Line \#3 & 9 & 5 & 0.300 & {$[0.126,0.512]$} & 17 & 0 & 0.028 & {$[0.000,0.082]$} \\
\hline Line \#4 & 8 & 1 & 0.111 & {$[0.015,0.287]$} & 29 & 3 & 0.067 & {$[0.019,0.141]$} \\
\hline Line \#5 & 12 & 3 & 0.154 & {$[0.045,0.312]$} & 25 & 5 & 0.115 & {$[0.044,0.214]$} \\
\hline All lines & 55 & 24 & 0.223 & {$[0.151,0.304]$} & 110 & 24 & 0.113 & {$[0.075,0.157]$} \\
\hline \multicolumn{9}{|c|}{ Generation F5 } \\
\hline Line \#1 & 16 & 8 & 0.265 & {$[0.133,0.425]$} & 24 & 8 & 0.180 & {$[0.088,0.297]$} \\
\hline Line \#2 & 12 & 3 & 0.154 & {$[0.045,0.312]$} & 25 & 10 & 0.212 & {$[0.113,0.331]$} \\
\hline Line \#3 & 10 & 7 & 0.364 & {$[0.181,0.570]$} & 11 & 1 & 0.083 & {$[0.011,0.219]$} \\
\hline Line \#4 & 22 & 4 & 0.109 & {$[0.037,0.212]$} & 24 & 7 & 0.160 & {$[0.073,0.272]$} \\
\hline Line \#5 & 17 & 5 & 0.167 & {$[0.066,0.303]$} & 22 & 9 & 0.217 & {$[0.112,0.346]$} \\
\hline All lines & 77 & 27 & 0.179 & {$[0.124,0.243]$} & 106 & 35 & 0.168 & {$[0.112,0.346]$} \\
\hline
\end{tabular}

${ }^{\mathrm{a} N u m b e r}$ of individuals genotyped.

${ }^{\mathrm{b}}$ Number of $R$ alleles among the individuals tested.

${ }^{\mathrm{c}}$ Number of $C k 80$ alleles among the individuals tested. 
all lines. At generations F3 and F5, this frequency was significantly lower than the initial frequency in all lines except line \#3 (Table 1). We observed no further changes in $R$ allele frequency within lines between the F3 and F5 generations. None of the lines displayed a genetic structure significantly different from what was expected under Hardy-Weinberg equilibrium, but we found an excess of $R S$ individuals in all lines as $\hat{f}$, the $F_{i s}$ estimator of Weir and Cockerham (1984), was negative $(-0.500<\hat{f}$ $\leq 0.077$ ) at both the F3 and F5 generations (Table 2). These excesses were due to the proportion of $R R$ individuals being lower than that predicted under HardyWeinberg equilibrium. In fact, all the carriers of the resistance allele detected during the course of the experiment were $R S$; we recovered no $R R$ individuals.

Despite the small number of individuals in each of the lines, and the eventuality of genetic drift, the frequency of the $R$ allele did not differ significantly between lines (Table 1). This absence of genetic differentiation made it possible to pool the data of the five lines for each of these two generations. Hence, for all lines considered together, the frequency of the $R$ allele was 0.223 for the F3 generation and 0.179 for the F5 generation (Table 1). The $95 \%$ CI of these frequencies did not include the value of 0.500 corresponding to the initial frequency in the F0 generation (Table 1). The slight decrease in $R$ allele frequency between generations F3 and F5 was not significant (Table 1). Finally, after pooling the data, the estimator $\hat{f}$ was -0.271 for the F3 generation and -0.206 two generations later (Table 2), with a deviation from Hardy-Weinberg equilibrium of borderline significance for the F3 generation ( $P=0.051$, see Table 2$)$.

During the course of the experiment, the relative frequencies of the two $C k$ alleles $-C k 80$ and $C k 100$ - remained almost identical. Within lines, significant changes were observed only in line \#1. In this line, the frequency of the Ck80 allele was significantly higher in the F3 generation than in the F0 and F5 generations (Table 1). For all lines considered together, the relative frequency of Ck80 did not differ significantly between the F0 (8.7\%) and F3 (11.3\%) and F5 (16.8\%) generations (Table 1)

Table 2 Proportion of the three different genotypes at the resistance locus within each line and for all lines considered together at generations F3 and F5

\begin{tabular}{|c|c|c|c|c|c|c|}
\hline & \multirow[t]{2}{*}{$\mathrm{N}^{\mathrm{a}}$} & \multicolumn{3}{|c|}{ Genotype } & \multirow[t]{2}{*}{$\hat{f}^{\mathrm{b}}$} & \multirow[t]{2}{*}{ P-value } \\
\hline & & SS & RS & $\mathrm{RR}$ & & \\
\hline \multicolumn{7}{|c|}{ Generation F3 } \\
\hline Line \#1 & 13 & 0.384 & 0.616 & 0.000 & -0.412 & 0.244 \\
\hline Line \#2 & 13 & 0.462 & 0.538 & 0.000 & -0.333 & 0.499 \\
\hline Line \#3 & 9 & 0.444 & 0.556 & 0.000 & -0.333 & 1.000 \\
\hline Line \#4 & 8 & 0.876 & 0.124 & 0.000 & - & - \\
\hline Line \#5 & 12 & 0.750 & 0.250 & 0.000 & -0.100 & 1.000 \\
\hline All lines & 55 & 0.564 & 0.436 & 0.000 & -0.271 & 0.051 \\
\hline \multicolumn{7}{|c|}{ Generation F5 } \\
\hline Line \#1 & 16 & 0.500 & 0.500 & 0.000 & -0.304 & 0.513 \\
\hline Line \#2 & 12 & 0.750 & 0.250 & 0.000 & -0.100 & 1.000 \\
\hline Line \#3 & 10 & 0.300 & 0.700 & 0.000 & -0.500 & 0.220 \\
\hline Line \#4 & 22 & 0.818 & 0.182 & 0.000 & -0.077 & 1.000 \\
\hline Line \#5 & 17 & 0.706 & 0.294 & 0.000 & -0.143 & 1.000 \\
\hline All lines & 77 & 0.350 & 0.650 & 0.000 & -0.206 & 0.108 \\
\hline
\end{tabular}

${ }^{\mathrm{a}} \mathrm{N}=$ number of individuals genotyped

${ }^{\mathrm{b}} F_{i s}$ estimator of Weir and Cockerham (1984).

'Probability of deviation from Hardy-Weinberg equilibrium.

\section{Discussion}

Our results confirm that alleles conferring resistance to Bt poplars producing the Cry3Aa toxin were not rare in beetle populations in French poplar stands before the widespread use of $B t$ sprays or $B t$ poplar in the fields. Three parents of the 176 feral individuals screened for $B t$ resistance were heterozygous for the Cry3Aa resistance allele carried by the R\#60 strain. We estimated the frequency of the resistance allele in the Bar-le-Duc population at 0.0113 , with a $95 \%$ credible interval of $0.0031-$ 0.0247. This frequency is slightly, but not significantly higher than our estimate for another French natural population - the Vatan population - collected from a site more than $500 \mathrm{~km}$ away. Indeed, in an $\mathrm{F}_{2}$ screen, the frequency of the resistance allele in the Vatan population was estimated at 0.0037 , with a $95 \%$ credible interval of 0.00045-0.0080 (Génissel et al, 2003a). The joint $95 \%$ credible region for the two estimated allele frequencies shows that the present estimate is not statistically different from that from Génissel et al (2003a) Figure 1.

Frequencies of $B t$ resistance alleles as high as those reported here in $C$. tremulae have been reported in lepidopteran pest species such as $H$. virescens (Gould et al, 1997), P. xylostella (Tabashnik et al, 1997) and P. gossypiella (Tabashnik et al, 2000). The frequencies in these insects may have been high because of previous selection for resistance. For example, Hawaiian populations of $P$. xylostella were treated with $B t$ and $B t$ cotton fields were planted in Arizona some years before collection of the $P$. gossypiella populations in which $B t$ resistance were detected. Similarly, the high level of Bt resistance discovered by Janmaat and Myers (2003) in greenhouse populations of cabbage loopers, Trichoplusia ni, probably resulted from the massive treatment of this pest with $B t$ toxins. Conversely, populations of C. tremulae have never been exposed to any particular

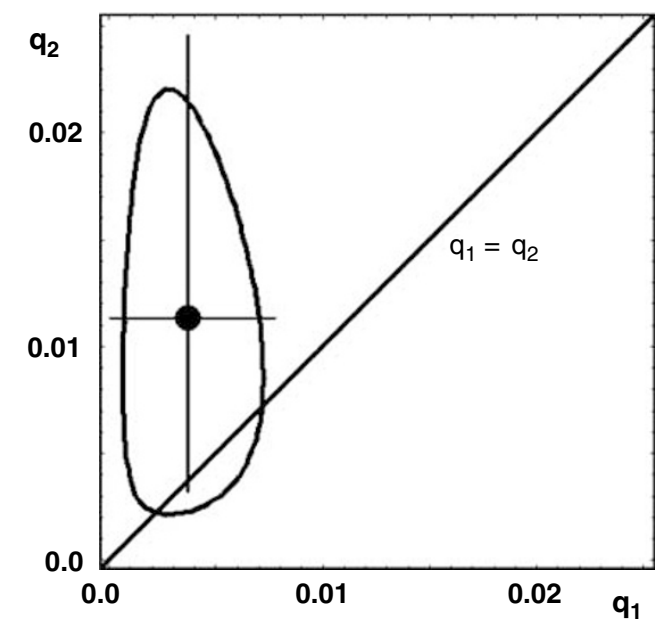

Figure 1 Joint 95\% credibility region for resistance allele frequency estimated by Génissel et al (2003a), $q_{1}$, and that estimated here, $q_{2}$. The diagonal line is the hypothesis $q_{1}=q_{2}$. The line intersects the 95\% credibility region. The point is the joint maximum likelihood estimate, $\left(\hat{q}_{1}, \hat{q}_{2}\right)$, and the lines going through the point are the $95 \%$ credibility intervals for each independent estimate of the allele frequency. 
selection pressure for $B t$ resistance due to human activities. Bt poplars have been planted only in a strictly protected insect-proof greenhouse and French poplar plantations, like other poplar plantations throughout Europe, have never been treated with Bt sprays. The results obtained in this study therefore support the conclusion of Génissel et al (2003a) that alleles conferring resistance to $B t$ plants may be present at detectable frequencies in pest populations before selection resulting from pest management by humans.

This conclusion is not restricted to $C$. tremulae. The frequency of an allele conferring a similar level of resistance ( $>5000$-fold) to the Cry3Aa toxin in the natural population from which Bauer (1995) selected her resistant strain of Chrysomela scripta was estimated at 0.0045 (Augustin et al, 2004). As in C. tremulae, this high frequency cannot have resulted from the application of $B t$ toxin on the host plant by humans. It would therefore be interesting to perform $\mathrm{F}_{2}$ screens on other coleopteran pests, such as Leptinotarsa decemlineata, Diabrotica virgifera virgifera, Phyllodecta vulgatissima and Phratora vitellinae, to determine whether a high level of Cry3Aa resistance is a general feature of the Chrysomelidae.

In C. tremulae, the high frequency of the Bt resistance allele over a large geographical area is consistent with the high rates of gene flow estimated by Génissel et al (2000). They showed, using allozyme markers, that there were no obvious limitations to gene flow between populations of $C$. tremulae over several hundreds of kilometres. They found no genetic differentiation $\left(F_{\mathrm{st}}=-0.001\right)$ between the Vatan population and the Contrexéville population, located at a site about $50 \mathrm{~km}$ south of Bar-le-Duc (Génissel et al, 2000). This intensive gene flow between populations may account for the similarity in the frequency of the $B t$ resistance allele at Vatan and Bar-le-Duc. We therefore pooled the Vatan and Bar-le-Duc estimates and calculate an expected $R$ allele frequency of 0.0049 , with a $95 \%$ credible interval of 0.0020-0.0091, as described by Stodola et al (2006). This allele would be expected to segregate at mutationselection balance rather than fluctuating at various frequencies following temporary and variable selection within poplar stands. The expected frequency at mutation-selection balance is about $u / h s$, with $u$ the mutation rate, $s$ the fitness cost and $h$ the dominance of this cost (Hartl and Clark, 1997).

Our study shows that $s$ is $>0$. Indeed, in the absence of selection on $B t$ poplar leaves, the frequency of the resistance allele decreased in all lines whereas allelic frequencies at the $C k$ allozyme locus - a neutral locus unlinked to the resistance locus - were unaffected. After three generations, for all lines considered together, the frequency of the resistance allele decreased from 0.500 to 0.223 , corresponding to a significant decrease of about 55\%. This frequency further decreased between the third and fifth generations, reaching 0.179 . The change in frequency was -0.044 (about a $20 \%$ decreased), but was not significant, due to the small number of beetles tested. Our results also suggest that $h<1$. None of the resistant individuals detected during the experiment was $R R$. There was therefore an excess of $R S$ individuals in all lines, this excess being marginally significant for all lines considered together. An incompletely dominant fitness cost would also account for the smaller decrease in allele frequency between the
F3 and F5 generations than between the F0 and F3 generations

This study provides further evidence that $B t$ resistance may entail a fitness cost in the absence of $B t$ selection. A decrease in resistance to $B t$ in $B t$-free environments has already been reported in laboratory strains of several pests (McGaughey and Beeman, 1988; Tabashnik et al, 1991; Janmaat and Myers, 2003). Unfortunately, the genetic basis of the resistance selected in these strains was generally unknown, as resistance ratio variation is generally investigated by assessing changes in $\mathrm{LC}_{50}$, and little attention was paid to the possible effects of genetic drift. Moreover, the initial resistance ratios of these strains were too low for these studies to be relevant for $B t$ crop management. Indeed, most of these resistant individuals were still susceptible to the doses produced by Bt crops. We show here that an allele conferring a sufficiently high level of resistance (resistance ratio $>5000$ ) for survival on Bt plants decreased in frequency in lines fed on non-transgenic plants. This decrease in frequency is probably due to fitness costs because genetic drift alone would be highly unlikely to give such results. This study provides no information about the life history traits potentially altered by the presence of this allele. Based on previous studies, the traits affected may be as different as overwintering success (Carrière et al, 2001b), fecundity and mating success (Groeters et al, 1994), larval survival (Carrière et al, 2005), maternal effects (Carrière et al, 2001a) and first-male paternity (Higginson et al, 2005).

Génissel et al (2003a) pointed out that Bt resistance allele frequencies exceeding $10^{-3}$ may result from a combination of a high mutation rate (eg $\left.u=10^{-5}\right)$ and a small (eg $s=0.01$ ) and/or recessive (eg $h=0.1$ ) fitness cost. Fitness cost cannot be determined directly from our data, but $s$ is most likely to be $>0.1$. If the frequency of the $R$ allele in the natural populations of $C$. tremulae were at mutation-selection balance, then the mutation rate would be at high but realistic values - between $10^{-4}$ and $10^{-7}$ - even with $0.1<s<0.5$ and $0.001<h<0.1$ (Table 3).

Our findings therefore provide both good and bad news for $B t$ resistance management. The bad news is that resistance allele frequency at the start of $B t$ plant cultivation may clearly be higher than originally thought, suggesting that resistance may be rapidly selected in target pests in the absence of refuges. The good news is that, like many other studies, this study shows that $B t$ resistance is counter-selected in Bt-free environments, making it possible to delay or to prevent the evolution of resistance if enough refuges are planted.

Table 3 Mutation rate $(u)$ estimated from the pooled frequency of the resistance allele $(p R)$, using various combinations of fitness costs $(s)$ and the level of dominance $(h)$ of these costs

\begin{tabular}{lccc}
\hline $\mathrm{pR}$ & $\mathrm{s}$ & $\mathrm{h}$ & $\mathrm{u}$ \\
\hline 0.0049 & 0.1 & 0.100 & $4.90 \times 10^{-5}$ \\
0.0049 & 0.1 & 0.050 & $2.45 \times 10^{-5}$ \\
0.0049 & 0.1 & 0.010 & $4.90 \times 10^{-6}$ \\
0.0049 & 0.1 & 0.005 & $2.45 \times 10^{-6}$ \\
0.0049 & 0.1 & 0.001 & $4.90 \times 10^{-7}$ \\
0.0049 & 0.5 & 0.100 & $2.45 \times 10^{-4}$ \\
0.0049 & 0.5 & 0.050 & $1.22 \times 10^{-4}$ \\
0.0049 & 0.5 & 0.010 & $2.45 \times 10^{-5}$ \\
0.0049 & 0.5 & 0.005 & $1.22 \times 10^{-5}$ \\
0.0049 & 0.5 & 0.001 & $2.45 \times 10^{-6}$ \\
\hline
\end{tabular}




\section{Acknowledgements}

We would like to thank L Leniaud, S Ponsard and C Vauvarin for help with electrophoresis and R Therene, $\mathrm{N}$ Millet for the multiplication of Bt poplars. This work was supported by the AO of the Ministere de la Recherche 'Impact des Organismes Génétiquement Modifés'.

\section{References}

Akhurst RJ, James W, Bird LJ, Beard C (2003). Resistance to the Cry1Ac $\delta$-endotoxin of Bacillus thuringiensis in the cotton bollworm, Helicoverpa armigera (Lepidoptera: Noctuidae). J Econ Entomol 96: 1290-1299.

Alstad DN, Andow DA (1995). Managing the evolution of insect resistance to transgenic plants. Science 268: 1894-1896.

Alyokhin A, Ferro DN (1999). Relative fitness of Colorado potato beetle (Coleoptera: Chrysomelidae) resistant and susceptible to the Bacillus thuringiensis Cry3A toxin. J Econ Entomol 92: 510-515.

Andow DA, Alstad DN (1998). F2 screen for rare resistance alleles. J Econ Entomol 91: 572-578.

Andow DA, Alstad DN (1999). Credibility interval for rare resistance allele frequencies. J Econ Entomol 92: 755-758.

Andow DA, Alstad DN, Pang Y-H, Bolin PC, Hutchinson WD (1998). Using an F2 screen to search for resistance alleles to Bacillus thuringiensus toxin in European corn borer (Lepidoptera: Crambidae). J Econ Entomol 91: 579-584.

Andow DA, Olson DM, Hellmich RL, Alstad DN, Hutchison WD (2000). Frequency of resistance to Bacillus thuringiensis toxin CryIAb in an Iowa population of European corn borer (Lepidoptera: Crambidae). J Econ Entomol 93: 26-30.

Augustin S, Courtin C, Réjasse A, Lorme P, Génissel A, Bourguet D (2004). Genetics of resistance to transgenic Bacillus thuringiensis Poplars in Chrysomela tremulae (Coleoptera: Chrysomelidae). J Ecol Entomol 97: 1058-1064.

Augustin S, Lévieux J (1993). Life history of the poplar beetle Chrysomela tremulae in the central region of France. Can Entomol 125: 399-401.

Bates SL, Zhao JZ, Roush RT, Shelton AM (2005). Insect resistance management in GM crops: past, present and future. Nat Biotechnol 23: 57-62.

Bauer LS (1995). Resistance: a threat to the insecticidal crystal proteins of Bacillus thuringiensis. Florida Entomol 78: 414-443.

Bentur JS, Andow DA, Cohen MB, Romena AM, Gould F (2000). Frequency of alleles conferring resistance to a Bacillus thuringiensis toxin in a Philippine population of Scirpophaga incertulas (Lepidoptera: Pyralidae). J Econ Entomol 93: 1515-1521.

Bird LJ, Akhurst RJ (2004). Relative fitness of Cry1A-resistant and -susceptible Helicoverpa armigera (Lepidoptera: Noctuidae) on conventional and transgenic cotton. J Econ Entomol 97: 1699-1709.

Bird LJ, Akhurst RJ (2005). Fitness of Cry1A-resistant and -susceptible Helicoverpa armigera (Lepidoptera: Noctuidae) on transgenic cotton with reduced levels of Cry1Ac. J Econ Entomol 98: 1311-1319.

Bourguet D, Chaufaux J, Séguin M, Buisson C, Hinton JL, Stodola TJ et al (2003). Frequency of alleles conferring resistance to Bt maize in French and US corn belt populations of the European corn borer, Ostrinia nubilalis. Theor Appl Genet 106: 1225-1233.

Brunk HD (1975). An Introduction to Mathematical Statistics. Xerox College Publishing: Lexington, KY.

Burd AD, Gould F, Bradly JR, Van Duyn JW, Moar WJ (2003). Estimated frequency of nonrecessive Bt resistance genes in bollworm, Helicoverpa zea (Boddie) (Lepidoptera: Noctuidae) in eastern North Carolina. J Econ Entomol 96: 137-142.

Carrière Y, Ellers-Kirk C, Biggs R, Degain B, Holley D, Yafuso C et al (2005). Effects of cotton cultivar on fitness costs associated with resistance of pink bollworm (Lepidoptera: Gelechiidae) to Bt cotton. I Econ Entomol 98: 947-954.

Carrière Y, Ellers-Kirk C, Liu Y-B, Sims MA, Patin AL, Dennehy TJ et al (2001a). Fitness costs and maternal effects associated with resistance to transgenic cotton in the pink bollworm. J Econ Entomol 94: 1571-1576.

Carrière Y, Ellers-Kirk C, Patin AL, Sims MA, Meyer S, Liu Y-B et al (2001b). Overwintering cost associated with resistance to transgenic cotton in the pink bollworm (Lepidoptera: Gelechiidae). J Econ Entomol 94: 935-941.

Carrière Y, Ellers-Kirk C, Sisterson M, Antilla L, Whitlow M, Dennehy TJ et al (2003). Long term regional suppression of pink bollworm by Bacillus thuringiensis cotton. Proc Natl Acad Sci USA 100: 1519-1523.

Carrière Y, Sisterson M, Tabashnik BE (2004). Resistance management for sustainable use of Bacillus thuringiensis crops in integrated pest management. In: Horowitz AR, Ishaaya I (eds) Insect Pest Management. Spring-Verlag: Berlin Heidelberg. pp 65-95.

Carrière Y, Tabashnik BE (2001). Reversing insect adaptation to transgenic insecticidal plants. Proc Roy Soc London B 268: $1475-1480$.

Coustau C, Chevillon C, ffrench-Constant R (2000). Resistance to xenobiotics and parasites: can we count the cost? Trends Ecol Evol 15: 378-382.

Ferré J, Van Rie J (2002). Biochemistry and genetics of insect resistance to Bacillus thuringiensis. Annu Rev Entomol 47: 501-533.

Génissel A, Augustin S, Courtin C, Pilate G, Lorme P, Bourguet D (2003a). Initial frequency of alleles conferring resistance to Bacillus thuringiensis poplar in a field population of Chrysomela tremulae. Proc Roy Soc London B 270: 791-797.

Génissel A, Leplé J-C, Millet N, Augustin S, Jouanin L, Pilate G (2003b). High tolerance against Chrysomela tremulae of transgenic poplar plants expressing a synthetic cry3aA gene from Bacillus thuringiensis ssp tenebrionis. Mol Breeding 11: 103-110.

Génissel A, Viard F, Bourguet D (2000). Population genetics of Chrysomela tremulae: a first step towards management of transgenic Bacilllus thuringiensis poplars Populus tremulae $\times P$. tremuloides. Hereditas 133: 85-93.

Georghiou GP, Taylor CE (1977). Operational influences in the evolution of insecticide resistance. J Econ Entomol 70: 653-658.

Gould F (1998). Sustainability of transgenic insecticidal cultivars: integrating pest genetics and ecology. Annu Rev Entomol 43: 701-726.

Gould F, Anderson A (1991). Effects of Bacillus thuringiensis and HD-73 Delta-endotoxin on growth, behavior, and fitness of susceptible and toxin-adapted strains of Heliothis virescens (Lepidoptera: Noctuidae). Environ Entomol 20: 30-38.

Gould F, Anderson A, Jones A, Sumerford D, Heckel DG, Lopez $\mathrm{J}$ et al (1997). Initial frequency of alleles for resistance to Bacillus thuringiensis toxins in field populations of Heliothis virescens. Proc Natl Acad Sci USA 94: 3519-3523.

Griffits JS, Aroian RV (2005). Many roads to resistance: how invertebrates adapt to Bt toxins. BioEssays 27: 614-624.

Groeters FR, Tabashnik BE, Finson N, Marshall WJ (1994) Fitness costs of resistance to Bacillus thuringiensis in the Diamondback moth (Plutella xylostella). Evolution 48: 197-201.

Hartl DL, Clark AG (1997). Principles of Population Genetics, 3rd edn. Sinauer: Sunderland, MA.

Higginson DM, Morin S, Nyboer ME, Biggs RW, Tabashnik BE, Carrière Y (2005). Evolutionary trade-offs of insect resistance to Bacillus thuringiensis crops: fitness cost affecting paternity. Evolution 59: 915-920.

Janmaat AF, Myers J (2003). Rapid evolution and the cost of resistance to Bacillus thuringiensis in greenhouse populations of cabbage loopers, Trichoplusia ni. Proc Roy Soc London B 270 : 2263-2270.

Lenormand T, Raymond M (1998). Resistance management: the stable zone strategy. Proc Roy Soc Lond B 265: 1985-1990. 
McGaughey WH, Beeman RW (1988). Resistance to Bacillus thuringiensis in colonies of indianmeal moth ans almond moth (Lepidoptera: Pyralidae). J Econ Entomol 81: 28-33.

Oppert B, Hammel R, Throne JE, Kramer KJ (2000). Fitness costs of resistance to Bacillus thuringiensis in the Indianmeal moth, Plodia interpunctella. Entomol Exp Appl 96: 281-287.

Pasteur N, Pasteur G, Bonhomme F, Catalan J, Britton-Davidian J (1987). Manuel technique de génétique par électrophorèse des protéines. Lavoisier: Paris.

Raymond M, Rousset F (1995). GENEPOP (version 1.2): population genetics software for exact tests and eucumenicism. $J$ Hered 86: 248-249.

Shelton AM, Zhao J-Z, Roush RT (2002). Economic, ecological, food safety, and social consequences of the deployment of Bt transgenic plants. Annu Rev Entomol 47: 845-881.

Stodola TJ, Andow DA (2004). F2 screen variations and associated statistics. J Econ Entomol 97: 1756-1764.

Stodola TJ, Andow DA, Hyden AR, Hinton JL, Roark JJ, Buschman LL et al (2006). Frequency of resistance to Bacillus thuringiensis toxin Cry1 Ab in southern US corn belt population of European corn borer (Lepidoptera: Crambidae). J Econ Entomol 99: 502-507.

Tabashnik BE, Carrière Y, Dennehy TJ, Morin S, Sisterson M, Roush RT et al (2003). Insect resistance to transgenic Bt crops: lessons from the laboratory and the field. J Econ Entomol 96: 1031-1038.
Tabashnik BE, Finson N, Johnson M (1991). Managing resistance to Bacillus thuringiensis: lessons from the diamondback moth (Lepidoptera: Plutellidae). J Econ Entomol 84: 49-55.

Tabashnik BE, Liu YB, Finson N, Masson L, Heckel DG (1997). One gene in diamondback moth confers resistance to four Bacillus thuringiensis toxins. Proc Natl Acad Sci USA 94: 1640-1644.

Tabashnik BE, Patin AL, Dennehy TJ, Liu YB, Carrière Y, Sims MA et al (2000). Frequency of resistance to Bacillus thuringiensis in field populations of pink bollworm. Proc Natl Acad Sci USA 97: 12980-12984.

Tang JD, Gilboa S, Roush RT, Shelton AM (1997). Inheritance, stability, and lack-of-fitness costs of field-selected resistance to Bacilus thuringiensis in diamondback moth (Lepidoptera: Plutellidae) from Florida. J Econ Entomol 90: 732-741.

Trisyono A, Whalon ME (1997). Fitness cost of resistance to Bacillus thuringiensis in Colorado potato beetle Coleoptera: Chrysomelidae). J Econ Entomol 90: 267-271.

Weir BS, Cockerham CC (1984). Estimating F-statistics for the analysis of population structure. Evolution 38: 1358-1370.

Wolfram Research Inc. (1999). Mathematica 4.0.0.0. Champaign, IL.

Wu K, Guo Y, Lv N, Greenplate JT, Deaton R (2002). Resistance monitoring of Helicoverpa armigera (Lepidoptera: Noctuidae) to Bacillus thuringiensis insecticidal protein in China. J Econ Entomol 95: 826-831. 